\title{
Relationship between inbreeding depression and selfing: the case of intrafamily selection
}

\author{
DENIS COUVET* \& JOËLLE RONFORT† \\ Institut d'Ecologie, Case 237, Université de Paris 6, 4 Place Jussieu, 75252 Paris Cedex 05 and $\uparrow$ Centre Emberger, \\ CNRS, Route de Mende, BP 5051, 34033 Montpellier, Cedex, France
}

\begin{abstract}
The outcome of intrafamily selection on variation in the load from deleterious mutations and on the magnitude of inbreeding depression has been investigated through an analytical one-locus model of mutation-selection balance, in partially selfing and in partially parthenogenetic populations. It is shown that, in contrast with an ordinary selection model, when intrafamily selection is assumed, increasing selfing rates are associated with increasing frequencies of a recessive deleterious allele, as well as with increasing magnitudes of inbreeding depression. With the same selective regime, a similar behaviour could be observed for the frequency of a deleterious allele in the case of parthenogenesis. On the basis of these results, intrafamily selection could have important consequences on the evolution of reproductive systems.
\end{abstract}

Keywords: inbreeding depression, intrafamily selection, mutation load, selfing.

\section{Introduction}

Intrafamily selection refers to the selective regime under which all families contribute to the same number of adults in the next generation, or more generally this number is independent of parental genotypes (Nagylaki, 1992). The usual case where the fitness of an individual depends only on its genotype and not on the frequency of the other genotypes in its family will in the present paper be referred to as ordinary selection.

Intrafamily selection is used in plant (thinning) and animal breeding (litter culling) to reduce genetic drift (refs in King, 1965); it has been proposed for use in the conservation of endangered species for the same purpose (Borlase et al., 1992) and also to retard unwanted adaptation to captivity (Allendorf, 1993). Such a selective regime may occur in natural populations when embryonic deaths increase the probability of survival of their sibs in the same litter, or when the female is able to conceive sooner than if the implantation of the unique embryo had succeeded. These two phenomena are sometimes called 'reproductive compensation'. Finally, intrafamily selection can occur when density-dependent recruitment of juveniles and restricted dispersal of gametes and zygotes are

\footnotetext{
*Correspondence.

†resent address: INRA, 34130 Mauguio, France.
}

combined (Ronfort \& Couvet, 1995), conditions that are likely to occur in natural populations.

The outcome of selection can depend on the selective regime in action. Relative to the case of ordinary selection, in the case of intrafamily selection the rate of directional selection is slower (King, 1965); for recurrent mutations to deleterious alleles, the mutation load, estimated as the number of deaths to get rid of a deleterious mutation, is higher (Campbell, 1988) and the frequency of deleterious alleles at equilibrium is doubled (Couvet \& Ronfort, 1994).

The outcome of selection might especially be dependent on the selective regime when the reproductive system varies. The reason is that intrafamily variability decreases as the amount of selfing increases, and hence the efficiency of selection in the case of intrafamily selection. To determine how intrafamily selection affects the covariation between the mutation load and the selfing rate, we performed an analytical resolution of a one-locus model of deleterious mutations. In a second case, parthenogenesis is examined.

\section{General assumptions}

Consider a large hermaphroditic population with nonoverlapping generations, which is either partially self-fertilizing (case 1) or partially parthenogenetic (case 2). Family size limitation occurs before repro- 
Table 1 Frequencies of the different offspring depending on frequencies of parents, in the case of intrafamily selection

\begin{tabular}{lllll}
\hline & & \multicolumn{2}{l}{ Frequency of offspring within each family (after selection) } \\
\cline { 3 - 5 } Mating & $\begin{array}{l}\text { Frequency } \\
\text { of mating }\end{array}$ & $A A$ & $A a$ & $a a$ \\
\hline$A A \times A A$ & self. $x+(1-$ self $) x^{2}$ & 1 & 0 & 0 \\
$A A \times A a$ & $(1-$ self $) 2 x y$ & $1 /(2-h s)$ & $(1-h s) /(2-h s)$ & 0 \\
$A A \times a a$ & $(1-$ self $) 2 x z$ & 0 & 1 & 0 \\
$A a \times A a$ & self. $y+(1-$ self $) y^{2}$ & $1 /(4-2 h s-s)$ & $(2-2 h s) /(4-2 h s-s)$ & $(1-s) /(4-2 h s-s)$ \\
$A a \times a a$ & $(1-$ self $) 2 y z$ & 0 & $(1-h s) /(2-h s-s)$ & $(1-s) /(2-h s-s)$ \\
$a a \times a a$ & self. $z+(1-$ self $) z^{2}$ & 0 & 0 & 1 \\
\hline
\end{tabular}

duction. Let $A$ and $a$ be the two allelic forms at the considered locus. $A$ represents the wild-type form which confers the higher fitness, and $a$ is the deleterious form, generated from $A$ by mutation at a per generation rate $u$. The relative fitnesses of the three corresponding genotypes are given below:

$\begin{array}{lccc}\text { Genotype } & A A & A a & a a \\ \text { Relative fitness } & 1 & 1-h s & 1-s\end{array}$

where $s$ is the magnitude of the deleterious effect (in the present model, it means that within a given family, for every wild-type homozygote reproducing, $1-s$ homozygotes for the deleterious allele will reproduce). $h$ is the dominance of this deleterious effect.

\section{The case of selfing}

The frequency of a given outcross is equal to the product of the frequency of the two genotypes involved, maternal and paternal, weighted by the probability of outcrossing 1 -self. Depending on the genotype of the parent from which male and female gametes are produced, there can be one to three different genotypes in a progeny (see Table 1). As selection operates within families, the selective value of a genotype in a given family depends on the proportions of the different genotypes weighted by their selective values in the case of ordinary selection (see above). As a result, when only one genotype is present within a progeny, its selective value. in the case of intrafamily selection is 1 ; this is the case for genotype $A a$ within the cross $A A \times a a$ (see Table 1).

Because the Hardy-Weinberg distribution does not hold in the case of familial selection, it is necessary to work with genotypic frequencies of $(A A, A a, a a)$, which will be called, respectively, $(x, y, z)$ and considered at the adult stage, at reproduction.
In the next generation, the frequencies of $(A A, A a, a a)$ indicated by, respectively, $\left(x^{\prime}, y^{\prime}, z^{\prime}\right)$ are the following (interaction terms between mutation and selection can be neglected):

$$
\begin{aligned}
x^{\prime}= & (1-\text { self })\left[x^{2}+\frac{2 x y}{2-h s}+\frac{y^{2}}{4-2 h s-s}\right] \\
& + \text { self }\left[x+\frac{y}{4-2 h s-s}\right]-2 u x, \\
y^{\prime}=2(1-\text { self })[x z+y(1-h s) & \left.\left(\frac{x}{2-h s}+\frac{y}{4-2 h s-s}+\frac{z}{2-h s-s}\right)\right]
\end{aligned}
$$

$$
+\operatorname{self}\left[\frac{2 y(1-h s)}{4-2 h s-s}\right]-u y+2 u x
$$

and

$$
\begin{aligned}
z^{\prime}= & (1-\text { self })\left[z^{2}+(1-s)\left(\frac{y^{2}}{4-2 h s-s}+\frac{2 y z}{2-h s-s}\right)\right] \\
& +\operatorname{self}\left[z+\frac{y(1-s)}{4-2 h s-s}\right]+u y .
\end{aligned}
$$

Recurrence eqns $1-3$ can be used to obtain equilibrium, where $\left(x^{\prime}, y^{\prime}, z^{\prime}\right)=(x, y, z)$.

From (3) we have the following.

- For $\operatorname{self}=1$, at equilibrium $z=1$.

- For $\operatorname{self}<1$ :

- for $h+$ self $\rightarrow 0, z \approx \frac{1-s}{4-2 h s-s} y^{2}$, 
and combining eqns 2 and $3 a$ yields the quadratic equation

$\frac{s}{4-s} y^{2}-2 u=0$, whose solution is

$y \approx \sqrt{\frac{2 u(4-s)}{s}} ;$

- for $h+$ self $>0, \quad z \approx \frac{1-s}{4-2 h s-s} \frac{\text { self }}{1-\text { self }} y$,

and combining eqns 2 and $4 \mathrm{a}$ yields the quadratic equation

$$
\begin{aligned}
& 2 y^{2}(1-s e l f)\left[(1+\gamma)\left(\gamma+\frac{1-h s}{2-h s}\right)-\frac{\gamma(1-h s)}{2-h s-s}\right. \\
& \left.-\frac{1-h s}{4-2 h s-s}\right] \\
& +y\left[\frac{h s}{2-h s}(1-\text { self })+\operatorname{self} \frac{s}{4-2 h s-s}\right]-2 u=0 \\
& \left(\text { where } \gamma \approx \frac{1-s}{4-2 h s-s} \frac{\text { self }}{1-\text { self }}\right) \text {, }
\end{aligned}
$$

whose solution is

$$
y \approx \frac{2 u(2-h s)}{s\left[h+\operatorname{self}\left(\frac{2-h s}{4-2 h s-s}-h\right)\right]}
$$

(the solution of the quadratic equation $D x^{2}+B x+C=0$ between 0 and 1 is

$$
x=\frac{-B+\sqrt{B^{2}-4 D C}}{2 D} \approx \frac{-C}{B} \text { when } D C \ll B^{2} \text { ). }
$$

In the case of ordinary selection:

- for $h+$ self $\rightarrow 0, q \approx \sqrt{\frac{u}{s}}$

(e.g. in Hopf et al., 1988);

- for $h+$ self $>0, q \approx \frac{u}{s\left[h+\frac{\text { self }}{2-\text { self }}(1-h)\right]}$ (from eqns $6 \mathrm{~b}$ and 9 in Lande \& Schemske, 1985).

For a given set of values for $h, s$ and self, the frequency (q) of the deleterious allele $a,(q=y / 2+z)$, is higher with intrafamily selection than in the case of ordinary selection. For $h+$ self $\rightarrow 0$, comparison of expressions 4 and $4 c$ yields immediately this result. For self $>0$ it can be demonstrated analytically by comparing expressions $4 \mathrm{~b}$ and $4 \mathrm{~d}$.

Moreover, whereas the frequency of the deleterious allele $a$ decreases with the rate of selfing in the case of ordinary selection (see eqn $4 \mathrm{~d}$ ), in the present case the frequency of $a$ is higher for complete selfing (where it reaches its maximum value, $q=1$ ), than for complete outcrossing.

\section{Variation in the deleterious allele frequency with selfing}

From eqns 4 and $4 b$ :

$$
\begin{aligned}
& q=\frac{u(2-h s) \beta}{h s}\left(1+\alpha \frac{\text { self }}{1-\text { self }}\right) \frac{1}{\beta+\text { self }} \\
& \left(\text { where } \alpha=\frac{2(1-s)}{4-2 h s-s} \text { and } \beta=\frac{h(4-2 h s-s)}{2-4 h+2 h^{2} s}\right)
\end{aligned}
$$

The selfing rate for which $q$ is minimized, $S_{q}^{*}$, is such that

$$
\frac{\mathrm{d} q}{\mathrm{~d} s e l f}=0
$$

and

$$
\frac{\mathrm{d}^{2} q}{\mathrm{~d} s e l f^{2}}<0
$$

where the differentials are evaluated at $S_{q}^{*}$. $S_{q}^{*}$ is a solution of the quadratic equation:

$\operatorname{self}^{2}(1-\alpha)-2 . s e l f+1-\alpha \beta=0$.

- $S_{q}^{*}=0$, for $s \leq \frac{-b+\sqrt{b^{2}-4 d c}}{2 d}$

(with $d=h\left(\frac{1-h}{2}-h^{2}\right), b=\frac{1}{2}-h+4 h^{2}, c=1-3 h$ ); 
This means that the frequency of the deleterious allele, $a$, can be minimized for intermediate selfing rates.

\section{Variations in the mutation load with selfing}

The load, $L$, is defined as $L=h s y+s z$. Then

$L=\frac{u(2-h s) \beta}{h}\left(2 h+\alpha \frac{\text { self }}{1-\text { self }}\right) \frac{1}{\beta+\text { self }}$,

(with $\alpha$ and $\beta$ defined as in eqn 5).

Using differentials as previously, the selfing rate for which the load is minimized, $S_{L}^{*}$, is a solution of the quadratic equation:

$\operatorname{self}^{2}(2 h-\alpha)-4 h . s e l f+2 h-\alpha \beta=0$.

- $S_{L}^{*}=0$, for $s \leq \frac{-b+\sqrt{b^{2}-4 d c}}{2 d}$

(with $d=h\left(1-h-2 h^{3}-4 h^{4}\right), b=-h^{2}-16 h^{4}$, $\left.c=-h+8 h^{2}-16 h^{3}\right)$;

- otherwise, $S_{L}^{*} \approx \frac{2 h-\sqrt{\alpha[2 h+\beta(2 h-\alpha)]}}{2 h-\alpha}$.

The selfing rate that minimizes the load in the case of intrafamily selection differs from the selfing rate that minimizes the frequency of the deleterious allele, except when $s$ is small, where both are zero.

\section{Variations in inbreeding depression with selfing}

If we call $w_{\mathrm{s}}$ the average fitness of selfed progenies and $w_{\mathrm{x}}$ the average fitness of outcrossed progenies, then $w_{\mathrm{x}} \approx 1-2 h s(y / 2+z)$ (neglecting terms of the order of $\left.u^{2}\right), w_{\mathrm{s}}=1-h s y / 2-s(y / 4+z)$ (as defined in eqns $5 \mathrm{a}$ and $5 \mathrm{~b}$ in Charlesworth \& Charlesworth, 1987). Then the inbreeding depression, $\delta$, is $\delta=1-w_{\S} / w_{\mathrm{x}}$.

$w_{\mathrm{s}}$ and $w_{\mathrm{x}}$ can be written as $w_{\mathrm{s}}=1-\lambda_{\mathrm{s}}$ and $w_{\mathrm{x}}=1-\lambda_{\mathrm{x}}$, where $\lambda_{\mathrm{s}}$ and $\lambda_{\mathrm{x}}$ tend towards zero, so that $w_{\mathrm{s}} / w_{\mathrm{x}} \approx 1-\lambda_{\mathrm{s}}+\lambda_{\mathrm{x}}$, and then $\delta \approx \lambda_{\mathrm{s}}-\lambda_{\mathrm{x}}$.

$$
\begin{aligned}
\delta & \approx s(1-2 h)\left(\frac{y}{4}+z\right) \\
& =\frac{u(2-h s)(1-2 h) \beta}{h}\left(\frac{1}{2}+\alpha \frac{\text { self }}{1-\text { self }}\right) \frac{1}{\beta+\text { self }} .
\end{aligned}
$$

For moderate dominance, there is a slight variation in inbreeding depression for low selfing rates. Inbreeding depression is most dependent on the selfing rate when deleterious alleles are slightly deleterious (Fig. 1).

Using differentials, the selfing rate for which inbreeding depression is minimized, $S_{\delta}^{*}$, is a solution of the quadratic equation:

$$
\begin{aligned}
& \operatorname{self}^{2}\left(\frac{1}{2}-\alpha\right)-\text { self }+\frac{1}{2}-\alpha \beta=0 . \\
& \text { - } S_{\delta}^{*}=0, \text { for } s \leq \frac{-3+14 h-8 h^{2}}{6 h-h^{2}-2 h^{3}}
\end{aligned}
$$

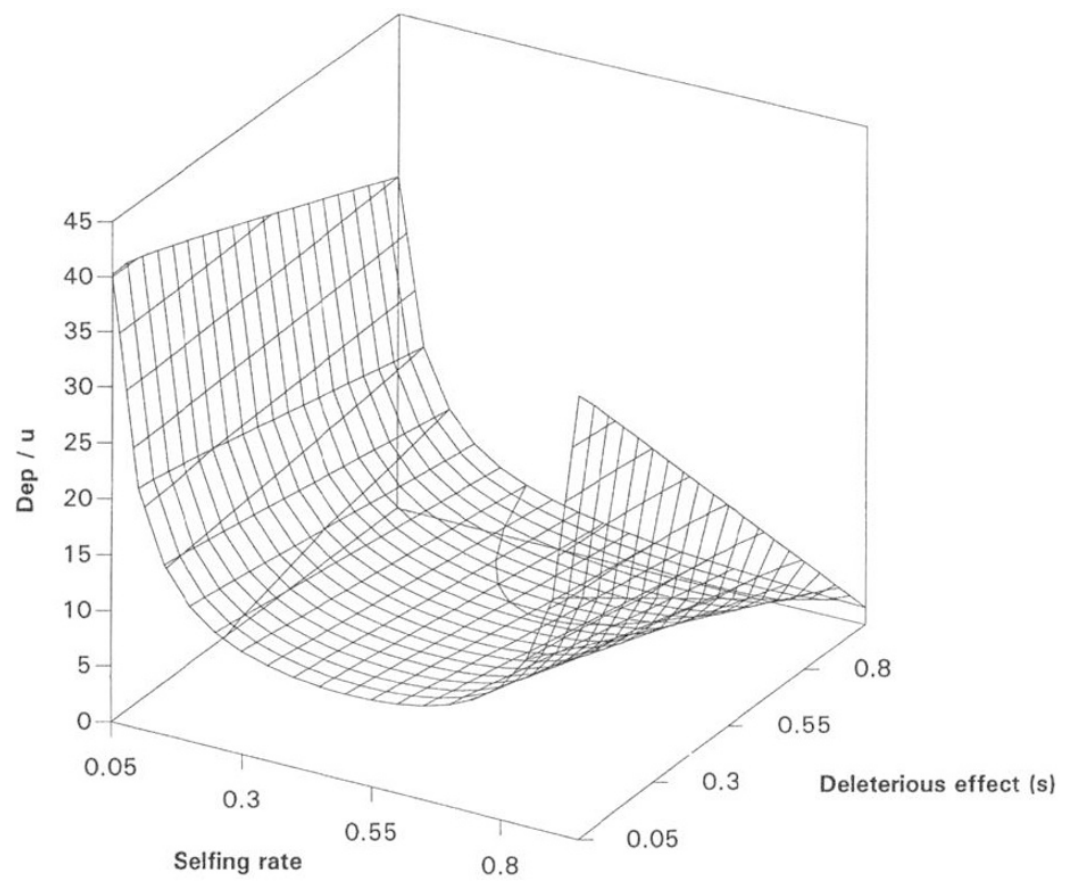

Fig. 1 Variation in the ratio inbreeding depression/mutation rate $(\operatorname{dep} / u)$, depending on selfing rate and magnitude of the deleterious effect (dominance, $h=0$ ). 
- otherwise, $S_{\delta}^{*} \approx \frac{\frac{1}{2}-\sqrt{\alpha\left[\frac{1}{2}+\beta\left(\frac{1}{2}-\alpha\right)\right]}}{\frac{1}{2}-\alpha}$.

With ordinary selection, inbreeding depression decreases with increasing selfing rates (Lande \& Schemske, 1985). Thus the relationship between selfing and inbreeding depression will be strongly affected by the selective regime. Inbreeding depression is minimized for complete selfing, i.e. as in the case of ordinary selection, only when mutations are very deleterious (i.e. $s$ is large, Fig. 2). This result can be explained as follows: when selection against deleterious mutations is strong ( $s$ is large), the fact that selection operates only within a family does not affect its impact. The selfing rate that minimizes inbreeding depression, $S_{\delta}^{*}$, decreases with $h$ and increases with $s$. Thus, inbreeding depression always increases with selfing for mutations that are mildly recessive and deleterious (Fig. 2).

For $h=0.2$ and $s=0.1$, values commonly assumed for deleterious alleles, based mainly on experiments on Drosophila (see discussion in Crow, 1993), $S_{\delta}^{*}$ reaches the value 0.2 ; hence intrafamily selection can potentially strongly affect the covariation of inbreeding depression with selfing rates, and consequently selection on selfing rates.

The two other minima, i.e. selfing rates that minimize the frequency of deleterious alleles and the load, respectively, show the same qualitative relationship with $h$ and $s$, that is they decrease with $h$ and increase with $s$. These three minima do not always coincide (Fig. 3).
For all values of $h$ and $s, S_{\delta}^{*} \leq S_{q}^{*}$. The value of $S_{L}^{*}$ relative to the two other minima depends on the value of $h$ :

for $h \leq \frac{1}{4} S_{L}^{*} \leq S_{\delta}^{*}$,

for $\frac{1}{4}<h<\frac{1}{2}, S_{\delta}^{*} \leq S_{L}^{*} \leq S_{q}^{*}$,

for $h>\frac{1}{2} S_{q}^{*} \leq S_{L}^{*}$.

\section{Selfing in the presence of biparental inbreeding}

As mentioned in the introduction, intrafamily selection can result from a combination of densitydependent recruitment and restricted dispersal of gametes and zygotes. In populations where restricted dispersal of gametes occurs, microdifferentiation of populations will lead to outcrossing events between genetically relatived individuals; such a mating pattern has been called 'biparental inbreeding' (Uyenoyama, 1986), to be distinguished from selfing (referred to as 'uniparental inbreeding' as only one parent is involved).

Biparental inbreeding and selfing will have identical genetic consequences, i.e. an increase in the frequency of homozygotes. We will call $r$ the probability, in the case of outcrossing, that identical genotypes mate, whereas $1-r$ is the probability that genotypes mate at random. In the present case there are two sources of inbreeding, selfing (frequency self) and biparental inbreeding (frequency $(1-$ self $) r)$. Thus for example the frequency of the cross $A a \times A a$ is self. $y+(1-$ self $) r y+(1-$ self $)(1-r) y^{2}$ (Table 2).
Fig. 2 Selfing rate that minimizes inbreeding depression $\left(S_{\delta}^{*}\right)$, depending on the dominance $(h)$ and the magnitude of deleterious effects $(s)$ of the deleterious allele $a$.

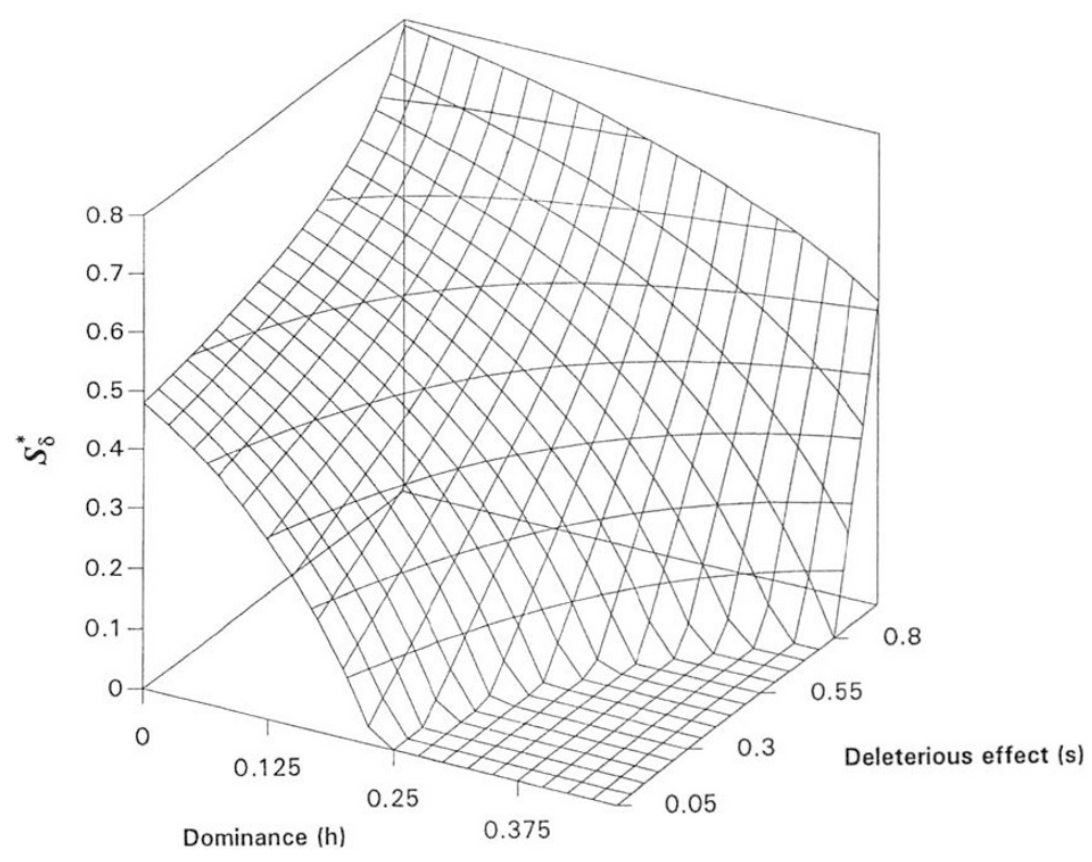




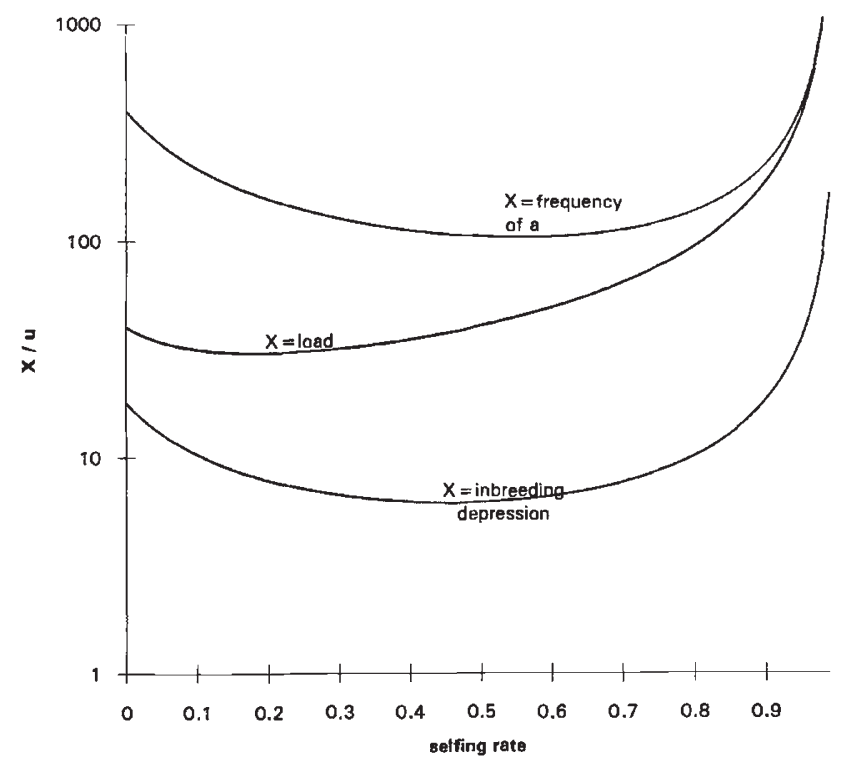

Fig. 3 Variation in the ratio of the frequency of the deleterious allele $a$, mutation load or inbreeding depression to the mutation rate $(x / u)$ with the selfing rate $(h=0.05, s=0.1)$.

Table 2 Frequencies of the different matings in the case of selfing and biparental inbreeding

\section{Frequency of mating under}

\begin{tabular}{llll}
\cline { 2 - 4 } Mating & Selfing & $\begin{array}{l}\text { Biparental } \\
\text { inbreeding }\end{array}$ & Outcrossing \\
\hline$A A \times A A$ & self.x & $(1-$ self $) r x$ & $(1-$ self $)(1-r) x^{2}$ \\
$A A \times A a$ & 0 & 0 & $(1-$ self $)(1-r) 2 x y$ \\
$A A \times a a$ & 0 & 0 & $(1-$ self $)(1-r) 2 x z$ \\
$A a \times A a$ & self.y & $(1-$ self $) r y$ & $(1-$ self $)(1-r) y^{2}$ \\
$A a \times a a$ & 0 & 0 & $(1-$ self $)(1-r) 2 y z$ \\
$a a \times a a$ & self. $z$ & $(1-$ self $) r z$ & $(1-$ self $)(1-r) z^{2}$ \\
\hline
\end{tabular}

In this case, recurrence equations for $(x, y, z)$ are:

$$
\begin{array}{r}
x^{\prime}=(1-\operatorname{self})\left[\begin{array}{l}
x^{2}+x r(y+z)+\frac{2 x y(1-r)}{2-h s} \\
\left.+\frac{y^{2}+y r(x+z)}{4-2 h s-s}\right]
\end{array}\right. \\
+\operatorname{self}\left[x+\frac{y}{4-2 h s-s}\right]-2 u x, \\
y^{\prime}=2(1-\operatorname{self})\left[x z(1-r)+y(1-h s)\left(\frac{x(1-r)}{2-h s}\right.\right.
\end{array}
$$

$$
\begin{array}{r}
\left.\left.+\frac{y+r(x+z)}{4-2 h s-s}+\frac{z(1-r)}{2-h s-s}\right)\right] \\
+\operatorname{self}\left[\frac{2 y(1-h s)}{4-2 h s-s}\right]-u y+2 u x
\end{array}
$$

and

$$
\begin{array}{r}
z^{\prime}=(1-\text { self })\left[z^{2}+z r(x+y)+y(1-s)\right. \\
\left.\times\left(\frac{y+r(x+z)}{4-2 h s-s}+\frac{2 z(1-r)}{2-h s-s}\right)\right] \\
+\operatorname{self}\left[z+\frac{y(1-s)}{4-2 h s-s}\right]+u y .
\end{array}
$$

At equilibrium we have the following.

From eqn $8, z \approx \frac{1-s}{4-2 h s-s} \frac{\text { self }(1-r)+r}{(1-\text { self })(1-r)} y$.

Equations 7 and 9 yield the following quadratic equation for $y$ :

$$
\begin{aligned}
& 2 y^{2}(1-\text { self })\left\{(1-r)\left[(1+\gamma)\left(\gamma+\frac{1-h s}{2-h s}\right)-\frac{\gamma(1-h s)}{2-h s-s}\right]\right. \\
& \left.-\frac{(1+r \gamma)(1-h s)}{4-2 h s-s}\right\} \\
& +y\left[\frac{h s}{2-h s}(1-\text { self })+\text { self } \frac{s}{4-2 h s-s}\right]-2 u=0 \\
& \left(\text { with } \gamma \approx \frac{1-s}{4-2 h s-s}-\frac{\text { self }+r(1-\text { self })}{(1-s e l f)(1-r)}\right) \text {. }
\end{aligned}
$$

Thus $y \approx \frac{2 u(2-h s)}{s\left[h+(\operatorname{self}(1-r)+r)\left(\frac{2-h s}{4-2 h s-s}-h\right)\right]}$

(with the same method of approximations as to obtain expression $4 b$ ).

It is then possible to compute the corresponding load and inbreeding depression. As in the previous case, selfing rates that minimize the frequency of the deleterious allele, the magnitude of inbreeding depression and the mutation load, depend on $h$ and $s$.

Using differentials as previously, and noticing that terms to differentiate take the following general

(C) The Genetical Society of Great Britain, Heredity, 76, 561-568. 
form:

$K\left[x+\alpha \frac{\operatorname{self}(1-r)+r}{(1-\operatorname{self})(1-r)}\right] \frac{1}{\operatorname{self}(1-r)+r+\beta}$

(with $x=1$ for $S_{q}^{*}, x=2 h$ for $S_{L}^{*}$, and $x=1 / 2$ for $S_{\delta}^{*}$, and where the constant $K$ depends on $h$ and $s$ but not on self).

These selfing rates are solutions of the quadratic equation:

$\operatorname{self}^{2}(1-r)^{2}(x-\alpha)-2$ self $(1-r)(x+r(\alpha-x))$

$$
+x(1-r)^{2}-\alpha \beta-\alpha r^{2}=0
$$

(one can notice that this equation corresponds, respectively, to $5 \mathrm{a}, 5 \mathrm{~b}$ and $5 \mathrm{c}$ when $r=0$ ).

The minima are:

- $S^{*}=0$, when $\frac{x-\sqrt{\alpha[x+\beta(x-\alpha)]}}{(x-\alpha)}<r$

- otherwise $S^{*} \approx \frac{x-\sqrt{\alpha[x+\beta(x-\alpha)]}}{(x-\alpha)(1-r)}-\frac{r}{1-r}$.

These minima always decrease with $r$; for $s=0.2$ and $h=0.1$, the selfing rate that minimizes inbreeding depression, which is 0.2 for $r=0$, becomes 0 for $r=0.2$. The present results, for the case of selfing with biparental inbreeding, show that the selfing rate for which inbreeding depression is minimized decreases (and hence is more different from the case of ordinary selection) when restricted dispersal increases.

\section{The case of parthenogenesis}

We consider the case of parthenogenesis, i.e. the genotypes of offspring are the same as the genotypes of their parthenogenetic parents, with the exception of mutation events. part is the rate of parthenogenesis; each parent with a probability part yields progeny of the same genotype, and with a probability 1 -part will outcross. The frequencies of the different outcrossing patterns and the corresponding progenies are given in Table $1($ self $=0)$.

The recurrence equations for the genotypic frequencies are as follows:

$x^{\prime}=(1-$ part $)\left[x^{2}+\frac{2 x y}{2-h s}+\frac{y^{2}}{4-2 h s-s}\right]+$ part. $x-2 u x$,

$y^{2}=2(1-$ part $)\left[x z+y(1-h s)\left(\frac{x}{2-h s}+\frac{y}{4-2 h s-s}\right.\right.$

$$
\left.\left.+\frac{z}{2-h s-s}\right)\right]+ \text { part. } y-u y+2 u x
$$

and

$$
\begin{aligned}
& z^{\prime}=(1-\text { part })\left[z^{2}+(1-s)\left(\frac{y^{2}}{4-2 h s-s}+\frac{2 y z}{2-h s-s}\right)\right] \\
& + \text { part. } z+u y \text {. }
\end{aligned}
$$

At equilibrium we have the following.

- For part $\rightarrow 1, z \rightarrow 1$.

- For part $<1, z \ll y$ :

$$
\begin{aligned}
& \text { - for } h>0, q \approx \frac{u(2-h s)}{h s(1-\text { part })} ; \\
& \text { - for } h \rightarrow 0, q \approx \sqrt{\frac{u(4-s)}{2 s(1-p a r t)}} .
\end{aligned}
$$

The frequency of deleterious alleles, and hence the load, always increases with the rate of parthenogenesis in the case of intrafamily selection. This result is in contrast to the case of ordinary selection, where the frequency of a deleterious allele does not depend on the rate of parthenogenesis (Hopf $e t$ al., 1988).

One can notice that when the rate of outcrossing decreases, intrafamily variability will be more affected in the case of parthenogenesis than in the case of selfing: parthenogenesis of heterozygotes $A a$ gives rise only to offspring $A a$, whereas in the case of selfing the three genotypes $(A A, A a, a a)$ appear at frequencies $(1 / 4,1 / 2,1 / 4)$, respectively, before selection.

\section{Concluding remarks}

These models have shown that intrafamily selection modifies the relationship between the magnitude of inbreeding depression and the selfing rate, and leads to the possibility of an evolutionary stability of intermediate selfing rates where inbreeding depression is minimized.

We examined selection on selfing rate in a multilocus model of inbreeding depression, where reproductive success of individuals was nearly independent of their mutation load, so that there was intrafamily selection on deleterious alleles. In that model this independence was the result of a combination of restricted gene flow and densitydependent recruitment. The selfing rate selected for in that model corresponds approximately to the 
selfing rate that minimizes inbreeding depression (Ronfort \& Couvet, 1995). It remains to explore the rate of parthenogenesis that would be selected for in such populations.

Finally, although equalization of reproductive success of individuals maximizes the retention of genetic variability in a population (Borlase et al., 1992), this selective regime should lead to an increase in the mutation load (Couvet \& Ronfort, 1994). The present results show that this effect will be most important in populations of selfers and parthenogens, and could then have important implications for the choice of conservation strategies for endangered species or germplasm collections.

\section{References}

ALLENDORF, F. W. 1993. Delay of adaptation to captive breeding by equalizing family size. Conserv. Biol., 7, 416-419.

BORLASE, S. C., LOEBEL, D. A., FRANKHAM, R., NURTHEN, R. K., BRISCOE, D. A. AND DAgGaRd, G. E. 1992. Modeling problems in conservation genetics using captive Drosophila populations. Consequences of equalization of family sizes. Conserv. Biol., 7, 122-131.

CAMPBELL, R. B. 1988. Mating structure and the cost of deleterious mutation: postponing inbreeding. J. Hered., 79, 179-183.
CHARLESWORTH, D. AND CHARLESWORTH, B. 1987. Inbreeding depression and its evolutionary consequences. Ann. Rev. Ecol. Syst., 18, 237-268.

COUVET, D. AND RONFORT, J. 1994. Mutation load depending on variance in reproductive success and mating system. In: Loeschke, V., Tomiuk, J. and Jain, S. K. (eds) Conservation Genetics, pp. 55-68. Birkhäuser Verlag, Berlin.

CRow, J. F. 1993. Mutation, mean fitness, and genetic load. In: Futuyma, D. F. \& Antonovics, J. A. (eds) Oxford Surveys in Evolutionary Biology, vol. 9, pp. 3-42. Oxford University Press, Oxford.

HOPF, F. A., MICHOD, R. E. AND SANDERSON, M. J. 1988. The effect of the reproductive system on mutation load. Theor. Pop. Biol., 33, 243-265.

KING, J. L. 1965 . The effect of litter culling - or family planning - on the rate of natural selection. Genetics, 51, 425-429.

LANDE, R. AND SCHEMSKE, D. W. 1985. The evolution of self-fertilization and inbreeding depression in plants. Evolution, 39, 24-40.

NAGYLAKI, T. 1992. Introduction to Theoretical Population Genetics, Spriner-Verlag, Berlin.

RONFORT, J. AND COUVET, D. 1995. A stochastic model of selection on selfing rates in structured populations. Genet. Res., 65, 209-222.

UYENOYAMA, M. K. 1986. Inbreeding and the cost of meiosis: the evolution of selfing in populations practising biparental inbreeding. Evolution, 20, 388-404. 